\title{
HIV diagnoses in Australia fall as clinicians embrace pre-exposure prophylaxis
}

\section{Nicholas A Medland \\ Senior researcher and Sexual health physician}

\section{Andrew E Grulich \\ Program head and \\ Professor \\ The Kirby Institute, University of New South Wales, Sydney}

\section{Keywords \\ antiretroviral drugs, HIV prevention, human immunodeficiency virus, pre-exposure prophylaxis}

Aust Prescr 2020;43:182-3 https://doi.org/10.18773/ austprescr.2020.048

Related article:

Prescribing pre-exposure prophylaxis for HIV
HIV prevention has been revolutionised by preexposure prophylaxis (PrEP) with antiretroviral drugs. Since its introduction in Australia, rapidly and at scale, HIV diagnoses have fallen dramatically.

PrEP involves at-risk HIV-negative individuals taking co-formulated tenofovir and emtricitabine. When exposure occurs, high intracellular drug concentrations inhibit viral replication and infection is prevented. PrEP is supported by the strongest possible clinical evidence and is now recommended by Australian guidelines.' It is subsidised by the Pharmaceutical Benefits Scheme (PBS) and can be prescribed by GPs using a streamlined authority. The prescription quantity is 30 tablets and two repeats which puts the patient into a three-monthly testing and prescription cycle. Patients with hepatitis B should be referred for specialist care as these drugs are also active against hepatitis $B$ and starting and stopping them may precipitate a flare.

PrEP adherence and efficacy are highly correlated. Daily administration of tenofovir with emtricitabine has been found to be safe and effective for HIV prevention. It is most effective when taken daily and continuously with studies observing close to $100 \%$ efficacy in adherent patients. ${ }^{2}$ Although efficacy is maintained for sexual exposure in gay and bisexual men who take four or more tablets per week, daily adherence is required for other population groups or types of exposure, including vaginal sex.

PrEP is easy to prescribe. Baseline evaluation (and monitoring) of patients includes establishing eligibility (medium to high HIV risk), testing for HIV and sexually transmitted infection, ${ }^{3}$ and determining estimated glomerular filtration rate (eGFR). Australian guidelines recommend that condom use to prevent sexually transmitted infection should be discussed with patients.

Draft Australian guidelines now also recommend on-demand or episodic PrEP in men who have sex with men with less frequent or intermittent sexual exposure. 'Patients take a loading dose of two tablets between 2 and 24 hours before their sexual contact and continue daily dosing after that for a minimum of two doses, or longer if there is ongoing sex. To date, on-demand PrEP has only been shown to be effective in men who have sex with men ${ }^{4}$ and is therefore not recommended in women (including transgender women), or transgender men. It is contraindicated in patients with hepatitis B.

Elimination of HIV transmission will require a high uptake of PrEP in gay and bisexual men. Australia is a global leader in the early, targeted, high-coverage roll-out of PrEP in this population. Demonstration studies were providing prophylaxis to more than 18,000 people nationally at the time of PBS listing in April 2018. ${ }^{5}$ In the first 18 months after listing, 29,543 individuals had filled one or more PrEP prescriptions. The effects have been dramatic. In New South Wales, where PrEP has been scaled-up very rapidly, it was associated with a rapid decline in HIV diagnoses, in particular new infections. ${ }^{5}$ Declines in newly diagnosed HIV cases have also now been observed nationally, from 1028 in 2015 to 838 in $2018 .^{6}$

Australia's success has occurred because of key enabling factors including:

- early PBS listing

- $\quad$ primary care clinicians ready to prescribe PrEP or keen to learn

- a highly motivated target community

- proactive state and commonwealth governments

- academic institutions ready to lead early PrEP demonstration studies

- robust surveillance systems.

HIV prevention has been revolutionised by PrEP together with the treatment-as-prevention approach. This involves people who test positive for HIV starting antiretroviral therapy so the risk of transmission to others is reduced to zero. While previously HIV prevention was only behavioural (i.e. condoms), PrEP and treatment-as-prevention add complementary clinical interventions.

However, new inequalities are emerging. Nationally over five years, new HIV diagnoses have declined by $44 \%$ in Australian-born men who have sex with men. However, diagnoses have not declined in men who have sex with men who were born overseas. This population now makes up approximately $50 \%$ of new diagnoses. ${ }^{6}$ In NSW, $66 \%$ of these men have resided in Australia for four years or less. ${ }^{7}$ Most newly arrived men who have sex with men are ineligible for Medicare because of their visa status and this has emerged as a key risk factor for HIV. ${ }^{8}$ 
Clinicians, and in particular GPs, can help to eliminate HIV transmission in Australia by identifying their patients who are at risk, particularly men who have sex with men. They should encourage them to have three-monthly testing for HIV and sexually transmitted infections and discuss, offer or start PrEP. The at-risk community exists in every town and city in the country. We will only be successful in eliminating HIV if all of these individuals have access to PrEP. $<$

Nicholas Medland has received research funding from Gilead. Andrew Grulich has received research funding and honoraria for educational presentations from Gilead.

\section{REFERENCES}

1. Australasian Society of HIV Viral Hepatitis and Sexual Health Medicine. PrEP guidelines update. Prevent HIV by prescribing PrEP. Sydney: ASHM; 2019. https://ashm.org.au/ resources/hiv-resources-list/prep-guidelines-2019 [cited 2020 Nov 1]

2. Owens DK, Davidson KW, Krist AH, Barry MJ, Cabana M, Caughey AB, et al.; US Preventive Services Task Force.

Preexposure prophylaxis for the prevention of HIV infection: US Preventive Services Task Force recommendation statement. JAMA 2019;321:2203-13. https://doi.org/10.1001/ jama.2019.6390

3. STIs in Gay Men Action Group. Australian sexually transmitted infection and HIV testing guidelines 2019: for asymptomatic men who have sex with men. Sydney: NSW Government STI Programs Unit; 2019. https://stipu.nsw.gov.au/wp-content/ uploads/STIGMA_Guidelines2019_Final-1.pdf [cited 2020 Nov 1]

4. Molina JM, Charreau I, Spire B, Cotte L, Chas J, Capitant C, et al.; ANRS IPERGAY Study Group. Efficacy, safety, and effect on sexual behaviour of on-demand pre-exposure prophylaxis for HIV in men who have sex with men: an observational cohort study. Lancet HIV 2017;4:e402-10. https://doi.org/10.1016/S2352-3018(17)30089-9
5. Grulich AE, Guy R, Amin J, Jin F, Selvey C, Holden J, et al.; Expanded PrEP Implementation in Communities New South Wales (EPIC-NSW) research group. Population-level effectiveness of rapid, targeted, high-coverage roll-out of HIV pre-exposure prophylaxis in men who have sex with men: the EPIC-NSW prospective cohort study. Lancet HIV 2018;5:e629-37. https://doi.org/10.1016/S2352-3018(18)30215-7

6. The Kirby Institute. National HIV notifications: Q1 2014-Q4 2018. Sydney: UNSW; 2019. https://kirby.unsw.edu.au/ sites/default/files/kirby/report/National-HIV-QuarterlyReport_2018-Q4.pdf [cited 2020 Nov 1]

7. Health NS. NSW HIV Strategy 2016-2020, Quarter 22019 , Data Report. https://www.health.nsw.gov.au/endinghiv/ Publications/q2-2019-nsw-hiv-data-report.pdf [cited 2020 Nov 1]

8. Medland NA, Chow EP, Read TH, Ong JJ, Chen M, Denham I, et al. Incident HIV infection has fallen rapidly in men who have sex with men in Melbourne, Australia (2013-2017) but not in the newly-arrived Asian-born. BMC Infect Dis 2018;18:410. https://doi.org/10.1186/s12879-018-3325-0 